\title{
EXTENSION OF HOLOMORPHIC MAPS
}

\author{
BY BERNARD SHIFFMAN ${ }^{1}$ \\ Communicated October 27, 1975
}

In this note we announce results on extending holomorphic maps into compact complex manifolds that satisfy certain curvature conditions. These results are analogous to theorems on extending holomorphic maps into manifolds with holomorphic sectional curvatures $\leqslant 0$ obtained by the author [5] and independently by Griffiths [3]. A similar type of result on meromorphic extension of equidimensional maps has been given by Griffiths [2, Theorem D].

Let $E$ be a hermitian holomorphic vector bundle on a complex manifold $M$. Let $R(v, \bar{w}, s, \bar{t})$ denote the curvature tensor for the hermitian connection on $E$, where $v, w \in E, s, t \in T=$ holomorphic tangent bundle of $M$. (Our sign convention is chosen so that if $E=T$, then $R(s, \bar{s}, s, \bar{s})$ is the usual holomorphic sectional curvature of a unit tangent vector $s$.) Let $\wedge_{k} T$ denote the bundle of holomorphic $k$-vectors on $M\left(\bigwedge_{1} T=T\right)$ and suppose $\wedge_{k} T$ is given a hermitian metric. For a simple vector $v \in \bigwedge_{k} T_{x}(x \in M)$, we let $\Sigma_{v}$ denote the $k$-dimensional subspace of $T_{x}$ associated with $v$, and we consider the hermitian form $R_{v}$ on $\Sigma_{v}$ given by

$$
R_{v}(s, \bar{t})=R(v, \bar{v}, s, \bar{t}) \quad \text { for } s, t \in \Sigma_{v} .
$$

We say that a complex manifold $M$ has property $H_{k}$ if $\wedge_{k} T$ carries a hermitian metric such that for all simple $k$-vectors $v, R_{v}$ has at least one nonpositive eigenvalue. Note that $M$ has property $H_{1}$ if and only if $M$ has a hermitian metric with holomorphic sectional curvatures $\leqslant 0$.

THEOREM 1. Let $1 \leqslant j<k<n$, and let $M$ be a compact complex manifold that has properties $H_{j}$ and $H_{k}$. Let $D$ be a domain with smooth boundary in $\mathbf{C}^{n}$. Let $p \in \partial D$, and suppose $D$ is strictly $(n-k)$-pseudoconcave at $p$ (i.e., if $D=\{u<0\}$, then the Levi form of $u$ restricted to the "holomorphic" tangent space of $\partial D$ at $p$ has at least $k$ negative eigenvalues). Then every holomorphic map $f: D \longrightarrow M$ extends holomorphically to a neighborhood of $p$.

The analogous result for $k=1$ is given in [5, Lemma 3] where $M$ is allowed to be complete instead of compact (see also [3]).

AMS (MOS) subject classifications (1970). Primary 32H99, 32D15, 53C55.

${ }^{1}$ Research partially supported by NSF Grant and by a Sloan Fellowship. 
COROllary 1. Let $M$ be as in Theorem 1, and let $K$ be a compact subset of a domain $D$ in $\mathbf{C}^{n}$ where $n>k$. Then every holomorphic map $f: D-K$ $\rightarrow M$ has a holomorphic extension to $D$.

We let $\Delta^{m}$ denote the unit polydisk in $\mathbf{C}^{m}$. We consider the Hartogs domain $H_{k}^{n}$ consisting of all points $z=\left(z_{1}, \ldots, z_{n}\right) \in \Delta^{n}$ such that

$$
\max \left(\left|z_{1}\right|, \ldots,\left|z_{k}\right|\right)>1-\epsilon \text { or } \max \left(\left|z_{k+1}\right|, \ldots,\left|z_{n}\right|\right)<\epsilon
$$

(where $0<\epsilon<1$ ).

COROLlARY 2. Every holomorphic map $f: H_{k}^{n} \rightarrow M$, where $M$ is as in Theorem 1, extends holomorphically to $\Delta^{n}$.

EXAMPLE 1. Let $D$ be a bounded symmetric domain, and let $\Gamma$ be a discrete group acting holomorphically and without fixed points on $D$ such that $D / \Gamma$ is compact (see [6, Chapter XI]). Give $D / \Gamma$ the Kähler metric induced from the Bergman metric on $D$, which has holomorphic sectional curvatures $<0$ and holomorphic bisectional curvatures $\leqslant 0$. Let $M_{0} \subset D / \Gamma \times Y$, where $Y$ is an arbitrary $(k-1)$-dimensional compact hermitian manifold. It follows that $M_{0}$ has property $H_{q}$ for $k \leqslant q \leqslant \operatorname{dim} M_{0}$. If $M$ is a small deformation of $M_{0}$, then $M$ also has property $H_{q}$ for $k \leqslant q \leqslant \operatorname{dim} M$.

The proof of Theorem 1 uses the following lemma, which is similar to the disk theorem in [5, Theorem 2].

LEMMA 1. Let $f_{m}: \Delta^{k} \rightarrow M$, for $m=1,2, \ldots$, be a sequence of holomorphic maps, where $M$ is a compact complex manifold satisfying property $H_{k}$. Suppose $\left\{f_{m}\right\}$ converges uniformly on compact subsets of $\Delta^{k}-\{0\}$ to a holomorphic map $f_{0}: \Delta^{k}-\{0\} \rightarrow M$. Then $f_{0}$ is meromorphic (in the sense of Remmert) at 0 .

The proof of Lemma 1 uses the techniques of [5] together with results of Stoll [8], Bishop [1], and the author [4] on recognizing analytic sets.

To prove Theorem 1 , we consider a sequence of $k$-disks in $D$ converging to a $k$-disk $\Delta$ in $D \cup\{p\}$ with center at $p$. Lemma 1 tells us that $f \mid \Delta$ is meromorphic at $p$. Let $\Delta^{\prime}$ be a $(j+1)$-disk in $\Delta$ with center at $p$. The conclusion of the theorem then follows from the result below, which shows that $f \mid \Delta^{\prime}$ is holomorphic at $p$.

THEOREM 2. Let $f$ be a meromorphic map from $\Delta^{k}$ into a complex manifold $M$ that satisfies $H_{k-1}$. If $f$ is holomorphic on $\Delta^{k}-\{0\}$, then $f$ is holomorphic on $\Delta^{k}$.

The method of proof of Theorem 2 is as follows: Let $h$ be the metric on $\bigwedge_{k-1} T$ that satisfies $H_{k-1}$. Suppose $f$ is not holomorphic at 0 . Let $G$ denote the normalization of the graph of $f$, let $\pi: G \rightarrow \Delta^{k}$ be the natural projection, 
and let $S=\pi^{-1}(0)$. Then $S$ has pure dimension $k-1$, by a result of Grauert and Remmert. We construct a nontrivial section $\Phi \in \Gamma\left(S_{0}, O\left(\left(\bigwedge_{k-1} T_{S}\right)^{q}\right)\right)$, where $S_{0}$ is a smooth, affine algebraic, Zariski-open subset of $S$, and $q$ is a positive integer. Let $u=\left\langle\left(\tau^{*} h\right)^{q}, \Phi \otimes \bar{\Phi}\right\rangle \in \mathcal{C}^{\infty}\left(S_{0}\right)$, where $\tau: S_{0} \rightarrow M$ is the natural projection. The section $\Phi$ is constructed so that $u$ vanishes at infinity. The curvative condition implies that the Levi form of $u$ has at least one nonnegative eigenvalue at each point of $S_{0}$, contradicting the fact that $u$ attains its maximum on $S_{0}$.

COROllaRY 3. Let $f$ be a meromorphic map from a domain $D \subset \mathbf{C}^{n}$ into a complex manifold $M$ that satisfies $H_{k-1}$. If the indeterminacy locus of $f$ has codimension $\geqslant k$, then $f$ is holomorphic.

Combining Corollary 3 with a result of Siu [7] on extending meromorphic maps, one has the following result.

Corollary 4. Let $D$ be a domain in $\mathbf{C}^{n}$ and let $A$ be an analytic set in $D$ of codimension $\geqslant k$, where $k \geqslant 2$. Let $M$ be a compact Kähler manifold that satisfies $H_{k-1}$. Then every holomorphic map $f: D-A \rightarrow M$ has a holomorphic extension to $D$.

It is well known that any meromorphic map from a domain $D$ into a projective algebraic manifold extends meromorphically to the envelope of holomorphy of $D$. This fact together with Corollary 3 yields the following result.

Theorem 3. Let $D, p$ be as in Theorem 1. Let $M$ be a projective algebraic manifold (or a Moishezon manifold) that satisfies $H_{k}$. Then every holomorphic map $f: D \rightarrow M$ extends holomorphically to a neighborhood of $p$.

Corollaries 1 and 2 are also valid if $M$ satisfies the hypotheses of Theorem 3. It is not known if the condition $H_{j}$ in Theorem 1 is necessary for general $M$.

EXAMPLE 2. Let $M$ be a $k$-dimensional projective algebraic manifold whose canonical bundle $K_{M}$ carries a metric with positive semidefinite curvature form. For example, let $M$ be a smooth hypersurface of degree $\geqslant k+2$ in $\mathrm{P}^{k+1}$. (If degree $M=k+2$, then $K_{M}$ is trivial.) Then $M$ satisfies $H_{k}$ and the extension result of Theorem 3 is valid for maps into $M$.

\section{REFERENCES}

1. E. Bishop, Conditions for the analyticity of certain sets, Michigan Math. J. 11 (1964), 289-304. MR 29 \#6057.

2. P. A. Griffiths, Holomorphic mapping into canonical algebraic varieties, Ann. of Math. (2) 93 (1971), 439-458. MR 43 \#7668.

3. - Two theorems on extensions of holomorphic mappings, Invent. Math. 14 (1971), 27-62. MR 45 \#2202.

4. B. Shiffman, On the removal of singularities of analytic sets, Michigan Math. J. 15 (1968), 111-120. MR $37 \# 464$. 
5. Extension of holomorphic maps into hermitian manifolds, Math. Ann. 194 (1971), 294-258. MR 45 \#598.

6. C. L. Siegel, Analytic functions of several complex variables, Institute for Advanced Study (lecture notes), 1948.

7. Y. T. Siu, Extension of meromorphic maps into Kähler manifolds, Ann. of Math. (to appear).

8. W. Stoll, Über die Fortsetzbarkeit analytischer Mengen endlichen Oberflächeninhaltes, Arch. Math. 9 (1958), 167-175. MR 21 \#729.

DEPARTMENT OF MATHEMATICS, THE JOHNS HOPKINS UNIVERSITY, BALTIMORE, MARYLAND 21218 